\title{
Neutrino Detection With CLEAN
}

\author{
D. N. McKinsey ${ }^{\mathrm{a}}$ and K. J. Coakley ${ }^{\mathrm{b}}$ \\ a Yale University, New Haven, CT 06511, USA \\ ${ }^{\mathrm{b}}$ National Institute of Standards and Technology, Boulder, CO 80305, USA
}

\begin{abstract}
This article describes CLEAN, an approach to the detection of low-energy solar neutrinos and neutrinos released from supernovae. The CLEAN concept is based on the detection of elastic scattering events (neutrino-electron scattering and neutrinonuclear scattering) in liquified noble gases such as liquid helium, liquid neon, and liquid xenon, all of which scintillate brightly in the ultraviolet. Key to the CLEAN technique is the use of a thin film of wavelength-shifting fluor to convert the ultraviolet scintillation light to the visible. This allows the same liquid to be used as both a passive shielding medium and an active self-shielding detector, allowing lower intrinsic radioactive backgrounds at low energies.

Liquid neon is a particularly promising medium for CLEAN. Because liquid neon has a high scintillation yield, has no long-lived radioactive isotopes, and can be easily purified by use of cold traps, it is an ideal medium for the detection of rare nuclear events. In addition, neon is inexpensive, dense, and transparent to its own scintillation light, making it practical for use in a large self-shielding apparatus. If liquid neon is used in CLEAN, the center of the full-sized detector would be a stainless steel tank holding approximately 135 metric tons of liquid neon. Inside the tank and suspended in the liquid neon would be several thousand photomultipliers.

Monte Carlo simulations of gamma ray backgrounds have been performed assuming liquid neon as both shielding and detection medium. Gamma ray events occur with high probability in the outer parts of the detector. In contrast, neutrino scattering events occur uniformly throughout the detector. We discriminate background gamma ray events from events of interest based on a spatial Maximum Likelihood method estimate of event location. Background estimates for CLEAN are presented, as well as an evaluation of the sensitivity of the detector for $p-p$ neutrinos. Given these simulations, the physics potential of the CLEAN approach is evaluated.
\end{abstract}

PACS: 14.60.Pq, 26.65.+t, 29.40.Mc, 95.35.+d

\footnotetext{
ઋ Corresponding author D.N. McKinsey. Email: daniel.mckinsey@yale.edu
} 
Keywords: extreme ultraviolet, liquid neon, neutrino, scintillation, wavelength shifter.

\section{CLEAN}

Just as new physics can be learned by building accelerators with high collision energies, new physics can also be learned by building underground detectors with high sensitivity to rare events, such as neutrino interactions, scattering of dark matter particles, and double beta decay.

This latter field is now the source of great excitement, as the recent results of the Super-Kamiokande, SNO, and KamLAND neutrino detectors have proven that neutrinos have mass[1,2,3]. This is the first substantial change in the Standard Model of particle physics in the last 20 years. In addition, new highly sensitive dark matter detectors, such as CDMS, EDELWEISS, and ZEPLIN, have driven down the limits on dark matter scattering cross-sections, bringing us significantly closer to theoretical predictions for supersymmetric dark matter, and therefore closer to testing one of the best explanations for the missing matter of the universe[4,5,6]. In our quest to learn more about neutrinos and other weakly interacting particles, it would be extremely valuable to have a better detector technology than is currently available. Ideally, this detector technology would simultaneously provide low radioactive backgrounds, a low energy threshold, and large detector mass at reasonable cost.

\subsection{Experimental design}

The following is a description of a scheme that meets all of these requirements, in which liquid neon is used as a detection medium. It is called CLEAN, stand-

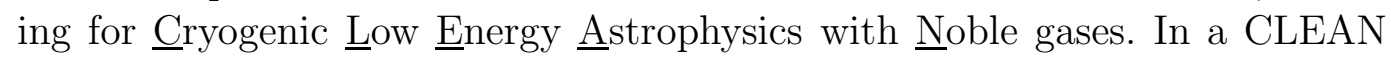
detector, neutrino-electron scattering events:

$$
\nu+\mathrm{e}^{-} \rightarrow \nu+\mathrm{e}^{-}
$$

and neutrino-nucleus scattering events:

$$
\nu+\mathrm{Ne} \rightarrow \nu+\mathrm{Ne}
$$


would be detected using liquid neon as a scintillator. ${ }^{1}$ The CLEAN concept was first proposed several years ago[7], and in the years following the basic approach has undergone some revision as new ideas have surfaced. Our current concept of the final CLEAN detector is shown in Figure 1.

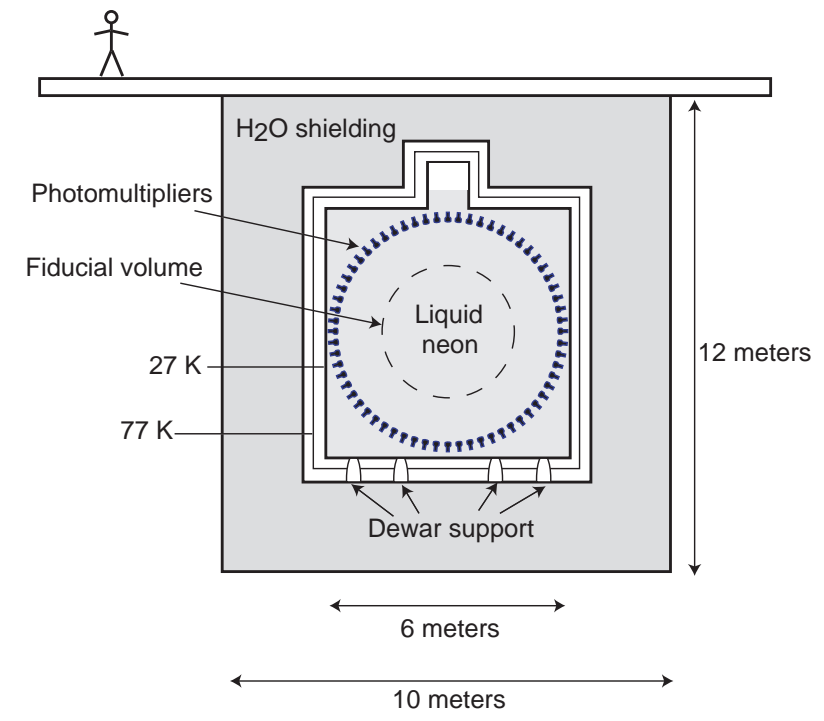

Fig. 1. A conceptual sketch of the full-sized CLEAN apparatus.

In this design, the central detector consists of a stainless steel tank filled with liquid neon. Supported by a spherical geodesic structure would be about two thousand photomultipliers facing the center of the tank. Each photomultiplier window would be coated with a thin layer of organic fluor material, which would convert the extreme-ultraviolet $(80 \mathrm{~nm})$ neon scintillation light to the visible. Only the central "fiducial volume" of the neon would be used for detecting neutrinos; the outer "shielding volume" would prevent gamma rays from the photomultipliers and tank from reaching the fiducial volume. Around the assembly would be a tank of water serving as gamma-ray shielding, neutron shielding, and muon veto.

Liquid neon has several characteristics that make it ideal for the detection of rare events:

- Like all of the liquified noble gases, liquid neon scintillates brightly in the hard UV. This translates into a large signal for ionizing radiation events $(15,000$ photons per $\mathrm{MeV})[8]$.

${ }_{1}$ While the CLEAN concept might be used with liquid helium or liquid xenon instead, this paper will concentrate on the liquid neon version. Liquid neon is significantly denser than liquid helium, while being easier to purify than liquid xenon, making it (in our view) especially promising for detection of rare events. 
- Like other noble liquids, liquid neon does not absorb its own scintillation light. The scintillation light results from the decay of neon excimers and is of lower energy than that needed to excite the ground-state neon atom. Scintillation light can therefore be extracted from the very large detectors that are necessary for the efficient detection of rare events.

- Neon has no long-lived isotopes and therefore has no inherent radioactivity. This stands in contrast to argon, krypton, and xenon, which contain natural radioactivity from the isotopes ${ }^{39} \mathrm{Ar},{ }^{85} \mathrm{Kr}$, and ${ }^{136} \mathrm{Xe}$. This also sets liquid neon apart from organic scintillator, which inevitably contains beta radiation from the decay of ${ }^{14} \mathrm{C}$.

- Neon has very low binding energies to a variety of surfaces. This allows neon to be very effectively purified of radioactive contaminants by use of cryogenic traps. Liquid helium at superfluid temperatures presents the only superior opportunity for cleaning contaminants [9].

- Liquid neon is dense $\left(\rho=1.2 \mathrm{~g} \cdot \mathrm{cm}^{-3}\right)$ and therefore uses space efficiently as a self-shielding medium. Liquid helium, in contrast, has a maximum density of $\rho=0.145 \mathrm{~g} \cdot \mathrm{cm}^{-3}$, and therefore requires more or different shielding. Liquid xenon has a density of $\rho=3.06 \mathrm{~g} \cdot \mathrm{cm}^{-3}$, and is even better at selfshielding, but at a greater cost and with likely greater contamination by impurities.

Though this approach requires a cryogenic apparatus (the boiling temperature of neon is $27 \mathrm{~K}$ ), this would not make the experiment overly complex or costly. For example, a commercial Gifford-McMahon cooler operating at $27 \mathrm{~K}$ with a cooling power of $75 \mathrm{~W}$ costs only $\$ 34,000$. With a total estimated heat load of $200 \mathrm{~W}$ in the full-sized CLEAN, the apparatus described above would derive little of its cost from its cooling requirements. The tank could be supported from below with stainless steel or titanium tubes, as is done commonly with large liquid helium-cooled accelerator magnets. Also, such a large cryogenic detector would not constitute a substantially new technical challenge; for example, the ICARUS collaboration has successfully built and tested a time projection chamber filled with 600 metric tons of liquid argon[10]. Also, the Fermilab 15-foot Bubble Chamber, filled with a neon-hydrogen mixture (61.7 $\%$ atomic neon, $38.3 \%$ atomic hydrogen), had a 23-ton mass when filled, and was successfully used for neutrino-electron scattering experiments[11].

\subsection{Scintillations in liquid neon}

A relatively clear model of scintillations in liquid neon can be elucidated from the numerous experimental characterizations of charged-particle-induced scintillation in condensed noble gases[12,13,14,15,16,17,18,19]. When an energetic charged particle passes through the liquid, numerous ion-electron pairs and

excited atoms are created. The ions immediately attract surrounding ground 
state atoms and form ion clusters. When the ion clusters recombine with electrons, excited diatomic molecules are created. Similarly, the excited atoms react with surrounding ground state atoms, also forming excited diatomic molecules. Fluorescence in condensed noble gases is observed to be almost entirely composed of a wide continuum of EUV light, emitted when these excited diatomic molecules decay to the monoatomic ground state. The energy of emission is less than the difference in energies between the ground state (two separated atoms) and the first atomic excited state for any given noble gas. The scintillation target is thus transparent to its own scintillation light, and a detector based on a condensed noble gas can be built to essentially arbitrary size without signal loss from reabsorption. The liquid will scatter its own scintillation light (a recent theoretical estimate predicts a Rayleigh scattering length of $60 \mathrm{~cm}[20]$ for liquid neon), but this light will eventually reach the walls of the detector and can be detected.

Recent studies of the scintillation properties of liquid neon have found it to be an efficient scintillation medium[8]. These measurements, when scaled to recent measurements of EUV scintillation yield in liquid helium, show that roughly 10,000 prompt EUV photons are emitted per MeV of electron energy. The wavelength of these photons was measured by Packard et al., who found that the electron-excited emission spectrum of liquid neon peaks at $80 \mathrm{~nm}[13]$. Liquid neon should also have an intense afterpulsing component due to the extreme ultraviolet radiation of triplet molecules. In liquid neon, the ground triplet molecular lifetime has been measured to be $2.9 \mu \mathrm{s}[8,21]$. The intensity of this afterpulsing is approximately $50 \%$ of the prompt signal, and can be added into the prompt signal to improve resolution of energy and position.

The liquid neon scintillation light cannot pass through standard window materials. Fortunately, recent work towards detection of ultracold neutrons trapped in liquid helium[22] has resulted in the characterization of efficient wavelength shifting fluors that convert EUV light into blue visible light[23]. This blue light is well matched to the peak sensitivity of available photomultiplier tubes. Tetraphenyl butadiene (TPB) is the fluor of choice, having a (prompt, $<20 \mathrm{~ns}$ ) photon-to-photon conversion efficiency from the EUV to the blue of roughly $100 \%[19,23]$. The prompt scintillation component from the combined liquid helium-waveshifter system has been measured to have a width of $20 \mathrm{~ns}$, allowing the use of coincidence techniques to reduce background[18]. In addition, as in organic scintillators, pulse-shape analysis could likely be used to discriminate light ionizers such as electrons from heavy ionizers such as alpha particles or nuclear recoils. Pulse shape analysis has proven to be effective in liquid xenon[24,25] and liquid helium[18], and we expect it to be effective in liquid neon as well. Combining the prompt and delayed components, one expects about 15,000 photons per $\mathrm{MeV}$ of electron energy. Given a waveshifting efficiency of $100 \%$, a photomultiplier covering fraction of $75 \%$, and a bialkali photocathode quantum efficiency of $15 \%$, a total photoelectron yield of about 
1690 per MeV could be achieved. With this photoelectron yield, the energy of a $100 \mathrm{keV}$ neutrino-electron scattering event could be measured with an average of 169 photoelectrons, with an associated 1-sigma random uncertainty of $7.7 \%$.

\subsection{External backgrounds}

We estimate the locations of ionizing radiation events in CLEAN by analyzing the pattern of photomultiplier hits [26]. The simulation consists of three steps. First, the external backgrounds (gamma and x-rays emitted from the photomultipliers) are simulated, and the locations of energy deposition for each gamma ray are recorded. Second, the trajectory of scintillation photons are simulated as they travel through the liquid,assuming a Rayleigh scattering length of $60 \mathrm{~cm}$ for liquid neon. When they enter the wavelength shifter, the photons are re-emitted isotropically (both toward and away from the closest photomultiplier), and the photons can be detected when they reach a photomultiplier (assuming a quantum efficiency of $15 \%$ ). The photomultiplier hit pattern due to each of these gamma rays is recorded. It is assumed that each photoelectron is recorded, so that each PMT can count multiple hits. Third, the event locations are estimated using a Maximum Likelihood method, yielding an estimated radius $r_{\text {est }}$ for the center-of-mass of energy deposition. The initial guess for the event location in the Maximum Likelihood method is the centroid of the multiple PMT photoelectron distribution.

We define an inner spherical fiducial volume $r \leq R p^{1 / 3}$ that occupies a fraction $p$ of the total spherical detection volume defined by $r \leq R$. In CLEAN the probability that an external background event occurs within the fiducial volume is low since most external gamma and x-rays do not penetrate into the inner fiducial volume. In contrast, events of interest occur uniformly throughout the entire detection volume. Thus, we can confidently discriminate external background events from events of interest that occur within a fiducial volume if we can accurately estimate the radial location of an event. We assign events into one of two classes. The first class consists of events that occur within the fiducial volume. The second class consists of events that occur outside the fiducial volume. The rate at which we assign external background events to the first class is the background rate for the CLEAN experiment. Following [26], we implement a classification rule based on the statistical distribution of $r_{\text {est }}$ determined from events that occur uniformly throughout the entire detection volume. In a Maximum Likelihood approach, we estimate the $p$ th quantile of the radial location estimates computed from the calibration data as a function of the number of detected photons. Events for which $r_{e s t}$ is less than this quantile are assigned to the first class. In [26], we also corrected $r_{\text {est }}$ using a polynomial calibration model to account for the effect of scattering 
and wavelength shifting.

To speed up our Monte Carlo simulation code, we modify the Maximum Likelihood approach as follows. We estimate the radial location of events in the calibration data set using a polynomial prediction model based on the centroid of the hit pattern ([26]). If this estimate exceeds $\beta R$ (where $\beta=0.8$ for the cases studied) we assign the event to the second class. Define the fraction of events for which the Centroid method estimates exceeds $\beta R$ to be $q$. For each event in the other $1-q$ fraction of the calibration data, we compute a Maximum Likelihood estimate $r_{e s t}$, and the $p /(1-q)$ th quantile of these estimates. Any event for which $r_{\text {est }}$ is less than this quantile is assigned to the first class.

Simulations have been performed for possible future CLEAN detectors of various sizes. The photomultipliers assumed for the simulations are multichannel plate photomultipliers, with assumed $U$ and Th levels of $3 \mathrm{ng} / \mathrm{g} .{ }^{2}$ All gamma and x-rays from $\mathrm{U}$ and $\mathrm{Th}$ with intensity greater than $1 \%$ were included. Figure 2 shows simulation results for several diameters of possible future CLEAN detectors. A fiducial volume corresponding to $r \leq 12.6 \mathrm{~cm}$ corresponds to a neon fiducial mass $10 \mathrm{~kg}$. The backgrounds arise from two sources: gamma rays that penetrate the shielding layer to deposit energy within the fiducial volume, and x-rays and low energy gammas that do not penetrate far and deposit only a small amount of energy. In the first case, there is little background at low energies, since low-energy gammas are very unlikely to penetrate the shielding layer. In the second case, there is little background at high energies, since high-energy events result in many scintillation photons, and the positions of these events can be accurately estimated. Simulation results for a full-size CLEAN detector have also been completed; the results are shown in Section 2.1, Figure 3 and compared with the expected solar neutrino signal.

The event reconstruction algorithm used in this study assumes that all detected photons produced by a particular event can be distinguished according to their arrival times from photon counts produced by other events. This is the only temporal information that we assume for event reconstruction. In contrast, in Super-K, SNO, KamLAND, and Borexino, event reconstruction algorithms are based on photon time-of-flight information. In the future, we plan to investigate the possibility of using additional photon time-of-flight information to improve the accuracy of our event reconstruction algorithm.

2 If it is determined that the optimal photomultipliers for CLEAN have somewhat higher levels of radioactivity, this will necessitate having a slightly larger liquid neon volume to achieve a given background level. However, as photomultipliers used in current neutrino experiments have U/Th levels on the order of 10-30 ng/g, and since the high-density liquid neon is an effective gamma attenuator, we expect the background levels indicated in Figures 2 and 3 to be reasonably accurate predictions. 


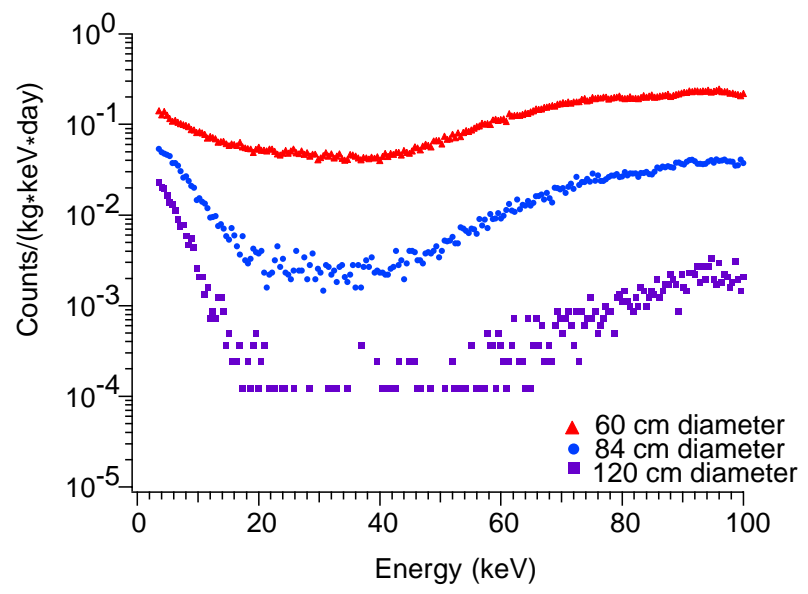

Fig. 2. External background rates in several sizes of future CLEAN detectors. Shown are estimated background rates for detectors of 60,84 , and $120 \mathrm{~cm}$ diameter, corresponding to neon masses of roughly 120,350 , and $1000 \mathrm{~kg}$. Results correspond to a fiducial volume of $r \leq 12.6 \mathrm{~cm}$, with a fiducial mass of $10 \mathrm{~kg}$.

\subsection{Internal backgrounds}

Because neon has a low binding energy on a variety of surfaces, we expect to be able to rid neon of all contaminants. These may be divided into two categories: EUV absorbers (such as $\mathrm{H}_{2}, \mathrm{~N}_{2}$, and $\mathrm{O}_{2}$ ) that could decrease the amount of detected EUV light, and radioactive impurities (such as Ar, $\mathrm{Kr}$, and $\mathrm{Rn}$ ) that could occasionally decay, creating unwanted backgrounds. ${ }^{39} \mathrm{Ar}$ is produced by muon spallation in the atmosphere and decays by $\beta^{-}$emission with an endpoint of $565 \mathrm{keV}$. The ratio of ${ }^{39} \mathrm{Ar} / \mathrm{Ar}$ has been measured to be $(8.1 \pm 0.3) \times 10^{-16}[27]$, and the full-size CLEAN requires a natural Ar level of less than $1 \times 10^{-10} \mathrm{~g} / \mathrm{g}$. ${ }^{85} \mathrm{Kr}$ is released from nuclear facilities as a fission product of ${ }^{235} \mathrm{U}$ and ${ }^{239} \mathrm{Pu}$. The ratio of ${ }^{85} \mathrm{Kr} / \mathrm{Kr}$ is roughly $1.5 \times 10^{-11}$, and the full-size CLEAN requires a natural Kr level of less than $5 \times 10^{-16} \mathrm{~g} / \mathrm{g}[28]$ to ensure that this background does not exceed $1 \%$ of the expected $p-p$ neutrino signal.

By passing the neon through cooled charcoal, all contaminants should be removed with high efficiency. The residence time for any species on a surface is proportional to $\exp \left(-E_{b} / k T\right)$. All of the contaminants have higher binding energies to charcoal than $\mathrm{Ne}$ (see Table 1), and at liquid neon temperature, $E_{b}>>k T$ for all possible contaminants of concern. A simple model of neon gas placed in contact with an activated charcoal-filled cold trap predicts a factor of $10^{6} \mathrm{Kr}$ reduction per stage.

Radon will not be an important background in CLEAN. As in the case of Ar and Kr, Rn will be removed by passing the Ne through cold traps. Also, because the diffusion of Rn through solid materials decreases markedly with 
Table 1

Binding energies $E_{b}$ of various atoms and molecules to charcoal surfaces. The well depth $D$ is also listed, since it approximates $E_{b}$ and is useful when $E_{b}$ has not been measured. "NM" denotes that no measurement is listed in reference [29].

\begin{tabular}{|c|c|c|c|c|c|}
\hline Species & $E_{b}(\mathrm{meV})$ & $D(\mathrm{meV})$ & Activity & $T_{1 / 2}$ & $Q(\mathrm{keV})$ \\
$\mathrm{Ne}$ & 30.1 & 32.6 & none & - & - \\
$\mathrm{H}_{2}$ & $41.6 \pm 0.3$ & $51.7 \pm 0.5$ & none & - & - \\
$\mathrm{O}_{2}$ & $\mathrm{NM}$ & 101.7 & none & - & - \\
$\mathrm{N}_{2}$ & $\mathrm{NM}$ & $104 \pm 3$ & none & - & - \\
$\mathrm{Ar}$ & $99 \pm 4$ & $96 \pm 2$ & ${ }^{39} \mathrm{Ar}\left(\beta^{-}\right)$ & $269 \mathrm{y}$ & 565 \\
$\mathrm{Kr}$ & 126 & $125 \pm 5$ & ${ }^{85} \mathrm{Kr}\left(\beta^{-}\right)$ & $10.76 \mathrm{y}$ & 687 \\
\hline
\end{tabular}

temperature, Rn will not enter the central tank through the walls. Any Rn originally in the neon will decay away with a half-life of 3.8 days and will be negligible within a month of running the experiment. In this connection, we note that the Borexino collaboration has demonstrated that by use of charcoal traps nitrogen gas can be purified to a Rn activity of less than $0.1 \mathrm{~Bq} \mathrm{~m}^{-3}$ in the gas[30]. Assuming that one can achieve equally low levels in neon (this is a conservative assumption because the binding energy of neon is less than that of nitrogen), the rate of $\mathrm{Rn}$ daughters decaying in CLEAN should be negligible.

\subsection{Cosmogenic activities}

The full-scale CLEAN experiment must be located in an underground facility to avoid radioactive backgrounds caused by muon spallation. While CLEAN will be surrounded by a muon veto to reject any prompt radioactivity, some isotopes will be created that decay with long half-lives and cannot easily be correlated with the muon pulses that created them. Many of the created nuclei decay via positron emission, and therefore deposit at least $1.022 \mathrm{MeV}$ in the neon, well above the energy window of interest. Others, such as ${ }^{14} \mathrm{C}$ and ${ }^{10} \mathrm{Be}$, decay with very long half-lives and are unlikely to create an appreciable background. This leaves ${ }^{23} \mathrm{Ne},{ }^{21} \mathrm{~F},{ }^{20} \mathrm{~F},{ }^{20} \mathrm{O},{ }^{19} \mathrm{O},{ }^{17} \mathrm{~N},{ }^{16} \mathrm{~N},{ }^{15} \mathrm{C},{ }^{11} \mathrm{Be},{ }^{7} \mathrm{Be}$, and ${ }^{3} \mathrm{H}$, all of which could create backgrounds in the energy range of interest. Most of these are beta emitters with endpoint energies of several MeV, and the majority of the decays will not create much background. One exception is ${ }^{7} \mathrm{Be}$; this decay has an endpoint of only $862 \mathrm{keV}$. Another exception is ${ }^{3} \mathrm{H}$ with an endpoint of $18 \mathrm{keV}$; however the energy deposited by this decay is rather small and most of these events will be below the CLEAN threshold.

While the cross-sections for the creation of these isotopes have not been mea- 
sured, we can roughly estimate their production rates based on careful studies of muon spallation cross-sections in ${ }^{12} \mathrm{C}$ and other nuclei. For example, the Borexino collaboration has measured a ${ }^{7}$ Be production cross-section of $230 \pm$ $23 \mu$ barn on ${ }^{12} \mathrm{C}$ at a muon energy of $190 \mathrm{GeV}[31]$. Conservatively assuming a much higher muon-neon spallation cross-section of 1 millibarn for the creation of each of the isotopes of concern, and assuming a muon flux of 0.23 $\mathrm{m}^{-2}$ day $^{-1}$ (the muon flux in SNOLAB), only 2.1 events per year would occur within the $10,000 \mathrm{~kg}$ fiducial volume and within the $0-800 \mathrm{keV}$ range of interest. If CLEAN were located at Gran Sasso, with a higher muon flux of $8.2 \mathrm{~m}^{-2}$ day $^{-1}$, then about 100 events per year would be expected within the energy window of interest.

Neutron absorption on $\mathrm{Ne}$ is not expected to contribute significant backgrounds. Both fast and slow neutrons, created largely from $\mathrm{U}(\alpha, n)$ reactions in the rock, will be absorbed by water shielding surrounding the liquid neon. Some high energy neutrons created by muon spallation may penetrate the water shielding, but at a deep site like SNOLAB the flux of these high energy neutrons is small. If neutrons are captured in the neon, then the unstable neon isotope ${ }^{23} \mathrm{Ne}$ (created by neutron absorption on ${ }^{22} \mathrm{Ne}$ ) can create decays in the energy range of interest. However, the neutron absorption cross-section of ${ }^{22} \mathrm{Ne}$ is small (46 mbarn for thermal neutrons), and the natural abundance of ${ }^{22} \mathrm{Ne}$ is only $9.2 \%$.

\section{The Physics Potential of CLEAN}

The CLEAN concept has multiple applications in detection of rare events. Here we discuss its application to four of them: $p-p$ neutrino detection, the search for the neutrino magnetic moment, and detection of supernova neutrinos.

\subsection{Solar neutrinos}

The study of neutrinos plays a prominent role in astrophysics and particle physics. Though they are emitted in vast numbers by stars and can be easily made in modern particle accelerators, neutrinos are difficult to detect because they have no charge and interact only through the weak force. Though the neutrino was first hypothesized in 1930 by Pauli to explain the observed characteristics of beta decay[32], it was many years later (1953) before the existence of the neutrino was confirmed experimentally by Reines and Cowan[33].

The dominant source of neutrinos is the Sun. While $90 \%$ of the Sun's energy is emitted as electromagnetic radiation, another $10 \%$ is emitted in the form 
of neutrinos[34]. And while the light takes about 10,000 years to diffuse out of the Sun, the neutrinos leave immediately. These neutrinos originate from a variety of nuclear reactions in the solar core known as the " $p-p$ chain".

In order to verify that the Sun is powered by nuclear fusion reactions, the first solar neutrino experiment (based on neutrino absorption by $\mathrm{Cl}$ ) was built in 1968 by Davis and collaborators[35]. While this experiment proved that the Sun is powered by nuclear reactions, the number of detected neutrinos fell well short of astrophysical predictions. In the years since this pioneering experiment, other detectors verified this shortfall. Two detectors based on neutrino absorption in Gallium, GALLEX [36,37] and SAGE[38,39], showed that the effect extended to low energy neutrinos, and the Kamiokande[40] and SuperKamiokande[42,43] detectors showed that the dearth of detected neutrinos extends across the entire solar neutrino spectrum. In addition, the sophistication of models of the solar interior have advanced, resulting in a sharper discrepancy with theory[44]. Therefore, recent years have seen a focusing of attention on the fundamental properties of the neutrino itself, since models that include neutrino mass and flavor mixing could cause the $\nu_{\mathrm{e}}$ emitted by the sun to be converted into $\nu_{\mu}$ and $\nu_{\tau}$, which cannot be absorbed by nuclei and have smaller cross-sections for neutrino-electron scattering.

The leading model for this flavor conversion is that neutrinos have mass, and that the neutrino mass matrix is non-diagonal when expressed in the flavor basis. The weak eigenstates $\left|\nu_{a}\right\rangle(a=\mathrm{e}, \mu, \tau)$ will be in this case linear superpositions of the mass eigenstates $\left|\nu_{i}\right\rangle(i=1,2,3)$, where $\left|\nu_{a}\right\rangle=\sum_{i} U_{a, i}\left|\nu_{i}\right\rangle$. If neutrinos have mass and mix according to this equation, then this results in neutrino oscillations; a neutrino that was initially in the weak eigenstate $a$ can be transformed into another weak eigenstate $a^{\prime}$. This oscillation has been observed for muon neutrinos, which change flavor after being created in the upper atmosphere (as shown by the Super-Kamiokande experiment[1]). Most recently, new results from SNO have proven decisively that solar neutrinos originally of the electron flavor are largely converted to $\mu$ or $\tau$ neutrinos by the time they reach the Earth $[2,45]$. The mechanism by which this oscillation occurs is believed to be the Mikheyev-Smirnov-Wolfenstein (MSW) effect, in which the neutrino states are mixed by matter interactions as they pass out of the Sun. In a simple $2 \times 2$ mixing scenario (approximately valid as long as $U_{e 3}$ is sufficiently small), one finds that the oscillation probability depends only on the difference $\Delta m^{2}=m_{i}^{2}-m_{j}^{2}$ between the squares of the mass eigenstates and one mixing angle $\theta$. The most heavily favored scenario is the MSW solution known as Large Mixing Angle, or LMA for short. The LMA solution has recently been confirmed by KamLAND[3], an experiment testing for oscillation of reactor antineutrinos.

Three compelling arguments yield the conclusion that the next large solar neutrino experiment should be designed to detect low energy solar neutrinos 
from the $p-p$ reaction, $p+p \rightarrow d+e^{+}+\nu_{e}$.

First, the $p-p$ neutrinos account for about $91 \%$ of the solar neutrino production according to the standard solar model (SSM). The resulting predicted flux at the Earth $\left(5.94 \times 10^{10} \mathrm{~cm}^{-2} \mathrm{~s}^{-1}\right)$ can be calculated to an uncertainty of $1 \%$. [44] As such, the $p-p$ flux is the most precisely predicted by the SSM. This spectacularly precise prediction calls out for an experiment to test it. Such a measurement would constitute an important test of the mechanism by which stars shine, with significant implications for all of astrophysics. An accurate $p-p$ neutrino experiment will also likely be able to measure the ${ }^{7} \mathrm{Be}$ neutrino flux. A measurement of the ratio of the $p-p$ flux to the ${ }^{7} \mathrm{Be}$ flux can determine the relative rates of the two termination reactions of the $p-p$ chain in the solar interior, ${ }^{3} \mathrm{He}-{ }^{3} \mathrm{He}$ and ${ }^{3} \mathrm{He}-{ }^{4} \mathrm{He}$, thus testing this prediction of the standard solar model[46].

Second, if the correct solution is LMA, then the neutrino $\Delta \mathrm{m}^{2}$ and $\tan ^{2} \theta$ parameters can be best determined with a combination of experiments. If the SSM is determined to be accurate, then a high-statistics $p-p$ neutrino experiment would yield a particularly good measurement of the mixing angle. This would nicely complement KamLAND, which will give an accurate measurement of $\Delta \mathrm{m}^{2}$. A measurement of the $p-p$ flux has an energy range very different from that of the ${ }^{8} \mathrm{~B}$ experiments, and uses neutrinos rather than the antineutrinos of KamLAND. It is also interesting to note that the LMA solution behaves qualitatively differently at low neutrino energies, where neutrino flavor conversion is dominated by vacuum oscillations, rather than by matterinduced oscillations as in the ${ }^{8} \mathrm{~B}$ neutrinos detected by Super-Kamiokande and SNO. Thanks to these differences, and thanks to the precise prediction of the $p-p$ neutrino flux, an accurate measurement of the $p-p$ flux would provide an independent test of the robustness of the neutrino mixing picture that is being assembled.

Third, a combination of low-energy experiments measuring different combinations of charged-current (CC) and neutral-current (NC) reactions would yield the total solar neutrino flux independent of flavor. By comparing this measured total flux to the predicted "standard candle" flux, tight limits can be placed on a possible sterile neutrino component. While SNO has shown that sterile neutrinos alone do not explain the solar neutrino problem, it nevertheless does not rule out a sizable sterile component[2]. The current $1 \sigma$ limit on an active-sterile admixture is $\sin ^{2} \eta \leq 0.16$, and the best chance for a significant improvement in sensitivity to sterile neutrinos is through the accurate measurement of the $p-p$ neutrino flux[46].

It is also likely that a new technology capable of a $p-p$ neutrino measurement could also measure the CNO neutrino flux through analysis of the neutrino spectrum in the energy window of 0.7 to $1.0 \mathrm{MeV}$ (above the ${ }^{7} \mathrm{Be}$ neutrino 


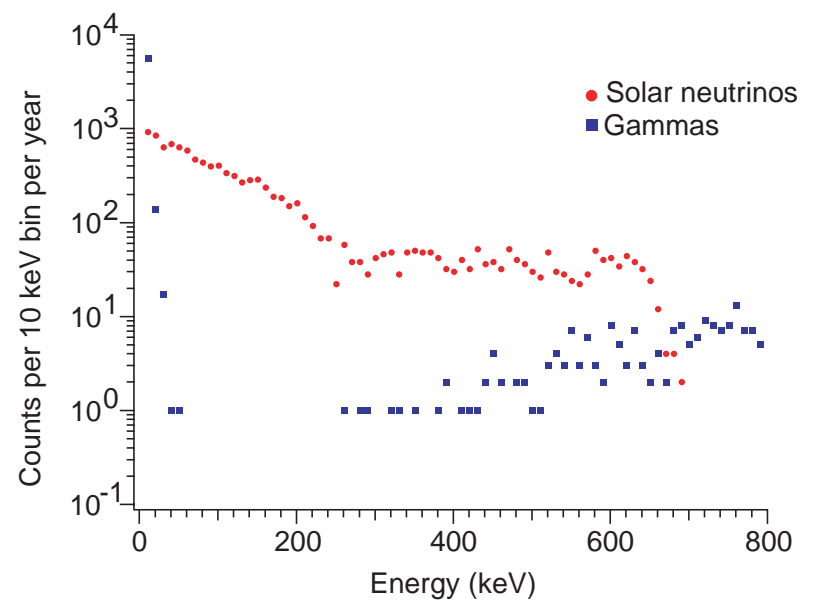

Fig. 3. Simulated neutrino and background rates in a CLEAN detector 6 meters in diameter, histogrammed versus energy. One year of simulation data are shown. Results correspond to a fiducial volume of $r \leq 125 \mathrm{~cm}$, and the data are allocated into $10 \mathrm{keV}$ bins.

scattering spectrum), provided that the detector is built to a sufficiently large scale. We have recently estimated the statistical uncertainty for such a measurement through Monte Carlo simulation[47] and conclude that for a CLEAN detector of $9 \mathrm{~m}$ diameter and 100 metric ton fiducial mass, the CNO flux could be measured with a statistical uncertainty of $13 \%$ with one year of data taking, assuming that the detector is deep enough that muon-induced backgrounds are not significant. There is currently no measurement of the CNO flux; the experimental upper limit on this flux is estimated to be $7.3 \%$ of the total solar neutrino flux[48].

We have investigated the ability of CLEAN to measure the p-p neutrinos through simulations of radioactive backgrounds, assuming that these backgrounds are dominated by gamma ray emission from the phototubes. Preliminary simulations have been performed for the full-size CLEAN, showing that for a neon tank of 6 meter diameter (135-ton total neon mass), these backgrounds are significantly smaller than the expected neutrino signal (about 2900 events per year assuming the LMA solution) in the energy range of interest (see Figure 3). As in the simulations of smaller detectors, the backgrounds at low energies are determined by position resolution, while the backgrounds at higher energies are dominated by gamma rays that manage to penetrate the shielding layer. Based on these simulations, a neutrino detection threshold of around $20 \mathrm{keV}$ or less is likely. 


\subsection{Neutrino magnetic moment}

As well as having masses, neutrinos could have magnetic moments. The best experimental limits on the magnetic moment of the neutrino come from experiments that look for deviations of the cross-sections for $\nu-e^{-}$and $\bar{\nu}-e^{-}$ scattering from those expected from the weak interaction alone. If the electron neutrino has a magnetic moment $\mu_{e}$, then it can scatter from electrons by magnetic dipole-dipole scattering, enhancing the scattering cross-section at low recoil energies. An additional term is then added to the scattering cross-section:

$$
\left(\frac{d \sigma}{d T}\right)_{\mu}=\frac{\pi \alpha^{2} \mu_{e}^{2}}{m_{e}^{2}} \frac{1-T / E_{\nu}}{T} .
$$

In this expression, $E_{\nu}$ in the initial neutrino energy and $T$ is the electron recoil energy. From this expression, it can be seen that if the neutrino has a magnetic moment, then the neutrino-electron scattering cross-section grows as $1 / \mathrm{T}$ at low recoil energies. Sensitivity to the neutrino magnetic moment can be enhanced by looking for neutrino-electron scattering events at low energy, where the electromagnetic scattering term is large. For this reason, it helps to have a low energy threshold when searching for a neutrino magnetic moment.

The lowest limits on the neutrino magnetic moments come from both solar neutrino experiments (which set limits on $\mu_{\nu}$ ) and reactor experiments (which set limits on $\mu_{\bar{\nu}}$ ). In the former category, Super-Kamiokande[43] has set the tightest limit to date, $\mu_{\nu}<1.5 \times 10^{-10} \mu_{B}$. In the reactor experiments, a limit of $1.3 \times 10^{-10} \mu_{B}(90 \%)$ was set by the TEXONO collaboration[49] using an ultra-low-background germanium counter, while the MUNU collaboration[50] has set a limit of $1.0 \times 10^{-10} \mu_{B}(90 \%)$ using a $\mathrm{CF}_{4}$-filled time-projection chamber. These two experiments had substantially different approaches. In the TEXONO experiment, a very low threshold was achieved, but internal backgrounds ultimately limited its sensitivity. In the MUNU experiment, very low backgrounds were achieved through a sophisticated gamma veto system and by comparing forward versus backward scattering rates. However, the threshold was much higher, about $600 \mathrm{keV}$.

Using a CLEAN detector, new limits could be placed on both $\mu_{\nu}$ and $\mu_{\bar{\nu}}$. First, a small (1 metric ton) liquid neon filled detector, placed near a nuclear reactor, would be very sensitive to neutrino-electron scattering, with a combination of low internal backgrounds and low threshold. With a 1-tonne detector, we estimate that the limit on $\mu_{\bar{\nu}}$ could be decreased by approximately two orders of magnitude. This experiment would have the additional bonus of measuring the low-energy neutrino flux from a reactor, something that has never previously been achieved. Second, by measuring the $p-p$ solar neutrino spectrum and comparing its spectral shape to that expected from the SSM and MSW oscillations, one may put a limit on the neutrino magnetic moment. Prelim- 
inary analysis shows that the full-size CLEAN, with a low energy threshold and low background rate, would be sensitive to a neutrino magnetic moment as low as $1 \times 10^{-11} \mu_{\mathrm{B}}$.

\subsection{Supernova neutrinos}

Following the gravitational collapse of a star with mass greater than eight solar masses, a type II supernova releases an energy of about $3 \times 10^{53}$ ergs in just a few seconds. Though supernovae can be seen visually, only $1 \%$ of the energy is emitted as light. The remainder is carried away by electron, mu, and tau neutrinos and antineutrinos, creating a large mixed neutrino flux at the Earth. Existing neutrino detectors such as Super-Kamiokande and SNO would easily detect these supernovae, just as SN1987A was detected by Kamiokande and IMB. However, these detectors do not yield much neutrino spectral information, and Super-Kamiokande is sensitive largely to electron anti-neutrinos and would give little information about the more energetic $\nu_{\mu}$ and $\nu_{\tau}$.

In a recent paper, we point out that the $\nu_{\mu}$ and $\nu_{\tau}$ neutrinos could be readily detected using a "flavor-blind" detector sensitive to neutrino-nuclear scattering[51], provided that it had a low energy threshold. With high sensitivity to neutral current interactions, such a low energy neutrino detector yields additional information about the dynamics of supernova collapse, the flavors of neutrinos emitted during a supernova, and the energy spectra of these neutrinos. Measurement of the $\nu_{\mu}$ and $\nu_{\tau}$ fluxes and energies would also give an accurate determination of the total energy released by the supernova. A similar approach is described in detail by Beacom et al., who discuss the possible detection of neutrino-proton scattering at KamLAND and Borexino[52].

Another approach to detecting heavy supernova neutrinos is through inelastic reactions on oxygen or carbon nuclei $[53,54,55]$. However, these reactions have relatively low yield, and neutrino energy information is lost since the outgoing neutrino is not detected.

The nuclear elastic scattering approach is promising because the coherent elastic scattering cross-section is large, and because all neutrino components contribute to the signal, allowing one to learn about $\nu_{\mu}$ and $\nu_{\tau}$ fluxes from the supernova. Assuming equal partitioning in energy among the $\nu_{e}, \bar{\nu}_{e}, \nu_{\mu}, \bar{\nu}_{\mu}, \nu_{\tau}$, and $\bar{\nu}_{\tau}$ components, we find that a standard supernova at 10 kiloparsecs gives a total yield of 3.99 events per metric ton in liquid Ne, with most of these (3.08) coming from $\nu_{\mu}, \bar{\nu}_{\mu}, \nu_{\tau}$, and $\bar{\nu}_{\tau}$. Because the supernova events occur within 10 seconds, a large fiducial volume could be used with very little background. For a 100 tonne fiducial volume, a total of $400 \nu$-Ne elastic events (285 above 
a $10 \mathrm{keV}$ threshold) could be detected from a supernova 10 kiloparsecs away.

The practical threshold attainable in CLEAN for supernova neutrinos depends on the quenching factor for nuclear recoils in liquid Ne. However, this factor should not be too small, as the denser liquid Xe has a quenching factor of $0.22 \pm 0.01[24]$. Though the quenching factor in liquid Ne has not yet been measured, one expects less quenching than the denser liquid Xe. Should a supernova occur, CLEAN could detect nearly all events above threshold, which could be as low as $4 \mathrm{keV}$.

\section{Ongoing research and development}

Before building a full-size CLEAN experiment, it makes sense to better study the scintillation and optical properties of liquid neon, test the scintillation response of liquid neon for nuclear recoils, build and test neon purification equipment, and test photomultipliers at low temperature. This is all part of the ongoing CLEAN research program.

One of the first projects is to build a $30 \mathrm{~kg}$ detector (micro-CLEAN). The liquid neon could be surrounded by as many as 10 photomultipliers, and much of the tank volume could be filled by acrylic or copper to reduce the total amount of neon to be liquified for a given test. A photomultiplier support structure will be built to allow various detector geometries. Using this apparatus, we intend to test:

- The light yield of liquid neon.

- Absorption and scattering of neon scintillation light.

- The coupling of the scintillation cell to neon purification systems.

- The performance of photomultipliers immersed in liquid neon.

- Methods of calibrating the detector for different radiation types.

- Pulse shape analysis for different radiation types.

- Development of calibrated event reconstruction algorithms for test geometry

Tests of scattering and absorption would be performed using collimated scintillation sources and photomultipliers in a carefully chosen geometry. The best geometry for these tests would be determined through Monte Carlo simulation. Based on previous measurements of scattering and absorption in organic scintillator done at the Borexino Counting Test Facility[56], we believe that a spherical geometry will be necessary to disentangle the effects of scattering and absorption. By placing a radioactive source inside a tube with one open end, while allowing the liquid neon to enter the tube, a collimated scintillation source can be created. Comparing the scintillation light detected by a photomultiplier facing the source to that detected by a photomultiplier that 
can detect only the scattered light, the scintillation scattering length can be measured. By comparing these measurements for different distances from the source to the photomultipliers, the absorption and scattering lengths can be determined independently.

A key component of CLEAN is the purification of liquid neon, removing elements that could absorb the EUV scintillation light or contribute to radioactive background. We expect to be able to rid neon of these contaminants by passing it through an adsorbant such as activated charcoal. In a working CLEAN experiment, two separate purification systems would be needed. The first purification system will use getters and liquid nitrogen-cooled charcoal traps to purify gaseous neon before liquification. The second purification system will be used to continuously purify liquified neon, removing liquid neon from the scintillation cell and returning it immediately, while allowing the liquid neon scintillation properties to be continuously monitored during purification. The apparatus would be housed in a bucket dewar and be surrounded by a liquid nitrogen bath. The system would use a cryorefrigerator to pull the liquid into a small chamber, then pressurize the liquid neon through a cold trap by closing the entrance valve and warming the neon. This approach has few moving parts and less opportunity for vacuum leaks to occur than if a mechanical pump were used to circulate the liquid neon. The system would make use of several cryogenic valves, which are available commercially. Two alternating cold traps would be used; while one purifies neon, the other is warmed and pumped out. The two neon purification systems have quite different technical requirements. The first system will be optimized for cleaning efficiency, but need not have high throughput, as the neon liquification rate will be limited by cooling power. The continous purification system will be optimized for neon flow rate, but need not have high purification efficiency. As the neon will be removed and replaced continuously, the best purification efficiency one can achieve is $1 / e$ per volume change.

We also intend to test a variety of photomultipliers at $27 \mathrm{~K}$ and to measure their radioactivity levels in order to determine the best light detector for use in CLEAN. Most photomultipliers used in nuclear and particle physics research are made with glass, which contains significant levels of radioactive U, Th, and K impurities. State-of-the-art photomultipliers with "ultralow background glass" have U and Th levels of about $30 \mathrm{ng} / \mathrm{g}$, and a K level of about $60 \mathrm{ng} / \mathrm{g}[57,58]$. However, it is quite possible that glass-less photomultipliers, with a mechanical structure composed of fused silica, metal, and sapphire, could have U, Th, and K levels on the order of 1 ppb. Though we would like to develop new photomultipliers that have low radioactive impurities and function at low temperature, photomultipliers that are currently on the market could be used as the photon detectors in CLEAN. Photomultipliers are currently being used by the ICARUS collaboration to detect scintillations in liquid argon $(90 \mathrm{~K})$, and we expect these to function at $27 \mathrm{~K}$ as well. 
To learn how sensitive CLEAN would be to nuclear scattering events, such as those arising from neutrino-nuclear scattering, the liquid neon scintillation should be calibrated with a neutron source. This procedure is common, especially for dark matter searches, and the approach in CLEAN would be similar to that followed in recent calibrations of liquid xenon-based detectors[24,25]. In order to perform this calibration, we would purchase a commercial neutron source that produces $2.5 \mathrm{MeV}$ neutrons via the $d+d \rightarrow n+{ }^{3}$ He reaction. We would detect scattered neutrons in coincidence with the flash of scintillation light from the liquid neon. By knowing the scattering angle $\theta$, the neutron energy, and amount of light detected, the experiment may be calibrated for nuclear recoils.

Excitation of the liquid neon with neutrons will also allow us to study the scintillation pulse shape from nuclear recoils in liquid neon. Pulse shape analysis has proven to be useful in experiments using liquid xenon as a target for WIMP detection[24,25], and we have previously studied liquid helium and found that it exhibits significantly different scintillation pulses for alpha and beta excitation[18,19]. A better understanding of how pulse shape varies with excitation density may allow us to also discriminate between radiation types in liquid neon. Given the very different times scales of triplet and singlet scintillation in liquid neon, it is likely that pulse shape discrimination in liquid neon will be quite effective.

\section{Conclusion}

The CLEAN approach to low background detection of rare events has potential application to low energy neutrino detection, supernova neutrino detection, and the search for the magnetic dipole moment of the neutrino. While the basic technique (use of a wavelength shifter and phototubes in combination with a liquified noble gas as detection medium) could in principle be used with liquid helium or xenon, liquid neon appears for the time being to be the most promising option. Ongoing research promises to determine liquid neon's light yield and pulse shape for a variety of radiation types, measure the optical properties of liquid neon for its own scintillation light, test photomultipliers immersed in liquid neon, and develop techniques for neon purification. There also remains work to be done on the event reconstruction algorithm, including empirical calibration of the event reconstruction algorithm, developing models for the transition matrix, possible incorporation of time-of-flight information, accounting for possible absorption effects, and development of approaches for cylindrical geometries. 


\section{Acknowledgements}

The authors thank A. Hime, E. Kearns, and T. Shutt for valuable discussions. The work of D. M. is supported by NSF grant PHY-0226142. Contributions of NIST (an agency of the US Government) to this work are not subject to copyright.

\section{References}

[1] Super-Kamiokande collaboration, Phys. Rev. Lett. 81 (1998) 1562.

[2] SNO collaboration, Phys. Rev. Lett. 87 (2001) 071371 and SNO collaboration, Phys. Rev. Lett. 89 (2002) 011301.

[3] KamLAND collaboration, Phys. Rev. Lett. 90 (2003) 021802.

[4] CDMS collaboration, Phys. Rev. D 68 (2003) 82002.

[5] Edelweiss collaboration, Phys. Lett. D 545 (2002) 43.

[6] S. Hart talk at DM2002, Marina del Rey.

[7] D. N. McKinsey and J. M. Doyle, J. Low Temp. Phys. 118 (2000) 153.

[8] R. A. Michniak, R. Alleaume, D. N. McKinsey, and J. M. Doyle, Nucl. Inst. and Meth. A. 482 (2002) 387.

[9] J. S. Adams et al., Proc. of the 8th Intern. Workshop on Low Temp. Detectors (LTD-8), Daltsen, NL (1999).

[10] ICARUS collaboration, Nucl. Inst. and Meth. A 508 (2003) 287.

[11] N. J. Baker, P. L. Connolly, S. A. Kahn, M. J. Murtagh, R. B. Palmer, N.P. Samios, M. Tanaka, C. Baltay, M. Bregman, M. Hibbs, M. Kalelkar, J. Okamitsu, and A. C. Schaffer, Phys. Rev. D 40 (1989) 2753 .

[12] M. Stockton, J.W. Keto, and W.A. Fitzsimmons, Phys. Rev. A 5 (1972) 372.

[13] R.E. Packard, F. Reif, and C. M. Surko, Phys. Rev. Lett. 25 (1970) 1435.

[14] C. M. Surko, R. E. Packard, G. J. Dick, and F. Reif, Phys. Rev. Lett. 24 (1970) 657.

[15] H. A. Roberts and F. L. Hereford, Phys. Rev. A 7 (1973) 284.

[16] S. Kubota, M. Hishida, M. Suzuki, and J.Ruan(Gen), Phys. Rev. B 20 (1979) 3486.

[17] A. Hitachi, T. Takahashi, N. Nobutaka, K. Masuda, J.Kikuchi, and T. Doke, Phys, Rev. B 27 (1983) 5279. 
[18] D. N. McKinsey, S. R. Brome, S. N. Dzhosyuk, R. Golub, K. Habicht, P. R. Huffman, E. Korobkina, S. K. Lamoreaux, C. E. H. Mattoni, A. K. Thompson, L. Yang, and J. M. Doyle, Phys. Rev. A 67 (2003) 062716.

[19] D. N. McKinsey, C. R. Brome, J. S. Butterworth, S. N. Dzhosyuk, R. Golub, K. Habicht, P. R. Huffman, C.E.H. Mattoni, L. Yanga, and J. M. Doyle, Nucl. Inst. and Meth. A 516 (2004) 475.

[20] G. M. Seidel, R. E. Lanou and W. Yao, Nucl. Inst. and Meth. A 489 (2002) 189.

[21] T. Suemoto and H. Kanzaki, J. Phys. Soc. of Japan 46 (1979) 1554.

[22] J. M. Doyle and S. K. Lamoreaux, Europhys. Lett. 26 (1994) 253.

[23] D. N. McKinsey, C. R. Brome, J. S. Butterworth, R. Golub, K. Habicht, P. R. Huffman, S. K. Lamoreaux, C. E. H. Mattoni, and J. M. Doyle, Nucl. Inst. and Meth. B 132 (1997) 351.

[24] D. Akimov, A. Bewick, D. Davidge, J. Dawson, A. S. Howard, I. Ivaniouchenkov, W. G. Jones, J. Joshi, V.A. Kudryavtsev, c, T.B. Lawsonc, V. Lebedenko, M. J. Lehner, P. K. Lightfoot, I. Liubarsky, R. Lscher, J. E. McMillan, C. D. Peak, J. J. Quenby, N. J. C. Spooner, T. J. Sumner, D. R. Tovey, C. K. Ward, Phys. Lett. B 524 (2002) 245.

[25] R. Bernabei, P. Belli, R. Cerulli, F. Montecchia, A. Incicchitti, D. Prosperi, C. J. Dai, M. Angelone, P. Batistoni, and M. Pillon, EPJdirect C11 (2001) 1.

[26] K. J. Coakley and D. N. McKinsey. Accepted to Nucl. Inst. and Meth. A.

[27] W. Kutschera, M. Paul, I. Ahmad, T. A. Antaya, P. J. Billquist, B. G. Glagola, R. Harkewicz, M. Hellstrom, D. J. Morrissey, R. C. Pardo, K. E. Rehm, B. M. Sherrill, and M. Steiner, Nucl. Inst. and Meth. B 92 (1994) 241.

[28] C. Y. Chen, Y. M. Li, K. Bailey, T. O'Connor, L. Young, and Z. T. Lu, Science 286 (1999) 1139.

[29] G. Vidali, G. Ihm, H.-Y. Kim, and M. W. Cole, Surface Science Reports 12 (1991) 133.

[30] Borexino collaboration, Astropart. Phys. 18 (2002) 1.

[31] T. Hagner, R. von Hentig, B. Heisinger, L. Oberauer, S. Schnert, F. von Feilitzsch and E. Nolte, Astropart. Phys. 14 (2000) 33.

[32] W. Pauli, 1930. Letter to the Physical Society of Tuebingen.

[33] F. Reines and C. Cowan, Phys. Rev. 90 (1953) 492.

[34] John N. Bahcall, "Neutrino Astrophysics", Cambridge University Presss, 1989.

[35] R. Davis, D. S. Harmer, and K. C. Hoffman, Phys. Rev. Lett. 20 (1968) 1205.

[36] GALLEX collaboration, Phys. Lett. B 285 (1992) 376. 
[37] GALLEX collaboration, Phys. Lett. B 447 (1999) 127.

[38] SAGE collaboration, Phys. Rev. Lett. 67 (1991) 3332.

[39] SAGE collaboration, Phys. Rev. C 60 (1999) 055801.

[40] Kamiokande collaboration, Phys. Rev. Lett. 65 (1990) 1297.

[41] kamiokande collaboration, Phys. Rev. Lett. 77 (1996) 1683.

[42] Super-Kamiokande collaboration, Phys. Rev. Lett. 82 (1999) 1810.

[43] Super-Kamiokande collaboration, Phys. Rev. Lett. 82 (1999) 2430.

[44] J. N. Bahcall, S. Basu, and M. H. Pinsonneault, Phys. Lett. B. 433 (1998) 1.

[45] SNO collaboration, nucl-ex/0309004

[46] J. N. Bahcall and C. Pena-Garay, hep-ph/0305159

[47] N. Ross, Statistical analysis of CNO neutrino detection, Junior Project, Princeton University Physics Department. Unpublished.

[48] J. N. Bahcall, M. C. Gonzalez-Garcia, and Pena-Garay, Phys. Rev. Lett. 90 (2003) 131301.

[49] H. T. Wong, Nucl. Phys. B 118 (2003) 454.

[50] MUNU Collaboration, Phys. Lett. B 564 (2003) 190.

[51] C. J. Horowitz, K. J. Coakley, and D. N. McKinsey, Phys. Rev. D 68 (2003) 23005 .

[52] J. F. Beacom, W. M. Farr, and P. Vogel, Phys. Rev. D 66 (2002) 033001.

[53] S. E. Woosley, D. H. Hartmann, R. D. Hoffman, W. C. Haxton, Astrophys. J. 356 (1990) 272

[54] A. Piepke, Nucl. Phys. B (proc. Suppl.) 91 (2001) 99.

[55] L. Cadonati, F. P. Calaprice, and M. C. Chen, Astroparticle Physics 16 (2002) 361.

[56] M. Johnson, Scintillator purification and study of light propagation in a large liquid scintillation detector, Ph. D Thesis, Princeton University. Unpublished.

[57] Electron Tubes, Inc., 100 Forge Way, Rockaway, NJ.

[58] Products or companies named here are cited only in the interest of complete description, and neither constitute nor imply endorsement by NIST or by the US government. Other products may be found to serve just as well. 